\title{
Menopause in Sri Lankan Context
}

\section{Silva $\mathbf{P}^{1}$, Moonesinghe $\mathbf{L}^{\mathbf{1}}$}

${ }^{1}$ Family Health Bureau, Ministry of Health, Sri Lanka.

Corresponding Author - Dr Padmaka Silva, Consultant Community Physician, Family Health Bureau

Email-padmakasilva@gmail.com

\section{Why menopause is important?}

The life expectancy of women is increasing worldwide due to the scientific and technological advances. It has been reported that the median age at menopause in Europe ranges from 50.1 to 52.8 years, in North America from 50.5 to 51.4 years, in Latin America from 43.8 to 53 years and in Asia from 42.1 to 49.5 years ${ }^{1}$. The average age of a Sri Lankan woman attaining menopause where cessation of menstrual bleeding lasts for at least one year, is between 49 and 51 years ${ }^{2,3}$. Considering the female life expectancy of 78 years, a woman has to spend approximately three decades of her life in the postmenopausal period. Therefore, in addition to being unable to bear children in post-menopausal period, overall health and wellbeing of middle-aged women has become a major global public health concem. However, there is a paucity of studies related to the menopausal symptoms and the QOL in pre and postmenopausal women in Sri Lanka ${ }^{2,3}$.

\section{Symptoms of menopause}

The main symptoms of menopause include vasomotor symptoms, problems related to changes in urogenital epithelium and those due to impaired cognitive functions. The sexual function of women is also affected due to these effects as well as other changes. Symptoms of menopause and their severity are different from person to per- son due to the effects of confounding factors ${ }^{4}$. Such confounding factors include lifestyle, social status, body composition, and psychological status $^{5}$. Menopausal symptoms, especially the vasomotor and sexual symptoms, are associated with impaired quality of life (QOL) in women ${ }^{3}$. QOL has been defined as "an individual's perception of their position in life in the context of the culture and value systems in which they live and in relation to their goals, expectations, standards and concerns"s. The QOL was significantly impaired among postmenopausal women [mean (SD);57.47(18.83)] compared to premenopausal women [mean (SD) ;66.82(17.93)].

\section{Challenges faced by postmenopausal women in Sri Lanka.}

New challenges in post-reproductive age women contribute significantly to the present day work force and some of them are in key positions by the time they reach menopause. Therefore the general wellbeing during this transition is important. Furthermore, nowadays women are becoming more and more socially active and engage in many physical activities and travelling, thus exposing themselves to risk of accidents and injuries. In societies such as Sri Lanka, it has been found that even older women who are not engaged in occupations remain active within their extended families, caring for grandchildren. All these demand a great deal of physical as well as psychological wellbeing. The increased life expectancy has increased the number of women in the post reproductive age who have non-communicable diseases. Oestrogen deficiency results in symptoms such as hot flushes, sweating, insomnia, vaginal dryness and discomfort ${ }^{2}$. Such symptoms can affect up to $85 \%$ of women. While symptoms tend to cease within five years in most, they can persist beyond that in a significant proportion. 


\section{Measures taken by the public health system in Sri Lanka}

Well Woman Clinics (WWC) were established in Sri Lanka in 1996. The program is delivered by the Medical Officers of Health and logistic and policy decisions are taken by the Well Woman Program in Family Health Bureau. There are approximately 1,000 clinic centres island wide. WWC- screens women aged 35 and 45 years for specific non communicable diseases, common female cancers and post reproductive health issues including menopause. At the WWC, women of 35 and 45 years of age are made aware of symptoms of menopause. Whenever necessary, women that attend WWC with menopausal symptoms are referred for specialized services through the Medical Officer of Health.

\section{Clinic attendance by clients aged 35 and $\underline{45}$ years}

There is a gradual increase in client turnover at WWC centers over the past few years. It is expected to increase the clinic attendance of women aged 35 years and women aged 45 years to $80 \%$ by year 2030 . Some districts have already reached $80 \%$ coverage for women aged 35 years. Health Education about symptoms of menopause is carried out by Medical Officers of Health, Public Health Nursing Sisters and Public Health Midwives at WWC.

\section{Service delivery in the curative sector}

Although the public health system which delivers WWC services has gradually expanded coverage and awareness of women of menopausal symptoms for women aged 35 and 45 years of age, curative services for those with such symptoms need to be expanded and streamlined.

\section{$\underline{\text { References }}$}

1) Palacios S, Henderson VW, Siseles N, Tan D, Villaseca P. Age of menopause and impact of climacteric symptoms by geographical region, Climacteric 2010; 13(5):419-428
2) Waidyasekera $\mathrm{H}$, Wijewardena $\mathrm{K}$, Lindmark G, Naessen T. Menopausal symptoms and quality of life during the menopausal transition in Sri Lankan women. Menopause 2009; 16(1):164-170

3) Goonaratna C, Fonseka P, Wijeywardene K. Perimenopausal symptoms in Sri Lankan women. Ceylon Medical Journal 1999; 44(2):63-69

4) Elsabaghand EEM, AbdAllah ES. Menopausal symptoms and the quality of life among pre/postmenopausal women from rural area in Zagazig city. Life Science Journal 2017; 9(2):283-291

5) Kothiyal P, Sharma M. Postmenopausal quality of life and associated factors - a review. American Journal of Pharm Research 2017; 3(9):7175-7183 